\title{
ESTIMATION OF VIBRIO SPECIES INCIDENCES AND ANTIBIOTIC RESISTANCE IN DIARRHEA PATIENTS
}

\author{
NUTAN THAKUR ${ }^{1}$, HARISH CHANGOTRA ${ }^{1}$, RAHUL SHRIVASTAVA ${ }^{1}$, NEELAM GROVER ${ }^{2}$, JITENDRAA VASHISTT ${ }^{1 *}$ \\ ${ }^{1}$ Department of Biotechnology and Bioinformatics, Jaypee University of Information Technology, Waknaghat, Solan, Himachal Pradesh, \\ India. ${ }^{2}$ Department of Pediatrics, Indira Gandhi Medical College, Shimla, Himachal Pradesh, India.
}

Email: jitendraa.vashistt@juit.ac.in

Received: 19 August 2017, Revised and Accepted: 10 November 2017

ABSTRACT

Objectives: Diarrheal diseases are globally important public health concern due to high morbidity and mortality rates among all age groups. Incidences and antimicrobial resistance patterns of Vibrio species are either underreported or overlooked in low-to-middle income countries. The present study is a hospital surveillance conducted to estimate the incidences of Vibrio infections in diarrhea.

Methods: A total of 572 diarrheal stool specimens were collected from two major hospitals and investigated using bacteriological tests coupled with molecular assays for characterization of Vibrio species. Kirby-Bauer's disk diffusion method was utilized to monitor antibiotic resistance against different antibiotic classes.

Results: Biochemical and molecular analysis of isolates revealed that Vibrio species accounted for about 5\% of diarrheal incidences. Higher rates of Vibrio spp. infections were observed among adolescent and adult age groups than children and elderly. Seasonal distribution analysis showed uniform Vibrio spp. infections throughout the year; however, frequency peaks were observed during rainy seasons. Vibrio spp. showed sensitivity for most antibiotics classes: Quinolones, aminoglycosides, and carbapenems; however, alarming resistance rates were observed against cephalosporins and nalidixic acid.

Conclusion: The present study provides credible estimates of Vibrio incidences and resistance pattern in diarrheal patients. Our findings will help in establishing trends in diarrhea etiology and management of severe diarrhea cases.

Keywords: Vibrio spp., Secretory diarrhea, Antimicrobial resistance, Childhood diarrhea, Seasonal variations.

(C) 2018 The Authors. Published by Innovare Academic Sciences Pvt Ltd. This is an open access article under the CC BY license (http://creativecommons. org/licenses/by/4. 0/) DOI: http://dx.doi.org/10.22159/ajpcr.2018.v11i1.22088

\section{INTRODUCTION}

Diarrhea is a multifactorial disease associated with numerous etiological agents which may cause high disease severity and persistence. A vast array of diarrhea associated pathogens includes bacterial, viral, and parasitic agents of which rotavirus and diarrheagenic Escherichia coli have been reported as main diarrheal pathogens among children [1]. Increased diarrhea incidences have been reported from the past decade, which implicated recurrent floods and natural catastrophe as main drivers of the communicable diseases [2]. Vibrio species signify as important public threat responsible for watery diarrhea in infected humans and are transmitted by fecal- oral route [3]. Globally, Vibrio is known to cause approximately three million cases per annum [4]. However, according to the World Health Organization (WHO), officially reported cases represent only 5-10\% of the total incidences [4]. Recent cholera outbreaks in Yemen alleged a very high morbidity and mortality incidences in the country [5]. Due to high morbidity and mortality rates associated with Vibrio spp. in epidemics and pandemics, continuous surveillance studies should be accelerated in developing nations. However, the national level surveillance for Vibrio species is limited to certain outbreaks in India, and there are no reports from most parts of the country [6].

Vibrio, member of family Vibrionaceae is a commashaped, Gram-negative, aquatic bacterium which colonizes small intestine of human [7]. Infections caused by Vibrio are typically categorized into Vibrio cholera and non-cholera Vibrio infections. Vibrio spp. causes profuse watery diarrhea and are historically known for worldwide epidemics and pandemics [8]. Symptoms of Vibrio-mediated gastroenteritis include rice watery stools, severe dehydration, fever, and vomit. Vibrio species express several virulence factors such as toxins (ctxA, stn, OmpW, and tox $R$ ), colonization factors (lipopolysaccharides, flagellar components, outer membrane proteins, hemagglutinins, and $\operatorname{tcp} A$ ), protease (hemolysins, cytolysins, thermolysins, and metalloproteases), and iron acquisition systems [9]. Cholera toxin encoded by ctxA gene has been isolated only from clinical strains and is utilized as an epidemiological marker to access toxigenic potential of Vibrio strains $[10,11]$.

Clinical guidelines for the treatment of diarrhea caused by Vibrio mainly focus on rehydration therapy and its maintenance. Probiotics and other traditional herbs are also utilized as traditional therapy $[12,13]$. However, use of antibiotics is mainly known to reduce severity and shedding of the pathogen [14]. Isolation of multidrug resistant Vibrio species from clinical sources is a major concern. Recent studies have reported a rise in antimicrobial resistance in Vibrio species against major classes of antibiotics (aminoglycosides, tetracyclines, and $\beta$-lactams) $[14,15]$.

The National Institute of Cholera and Enteric Diseases works as collaborating body with the WHO and depicted underreporting and negligence in cholera incidence surveillance [6]. Till date, seven cholera pandemics have been reported, and the Bay of Bengal was identified as an epicenter for the most recent one $[16,17]$. Molecular typing of Vibrio cholerae in nearby region of Himachal Pradesh revealed predominant ribotype IV and RIII $[18,19]$. Our previous study elucidated incidences of viral pathogens among moderate to severe cases [20]. The present research was performed as a part of the study to reveal Vibrio incidences and coinfection rates in diarrheal disease. Simultaneously, antibiotic resistance profiles of Vibrio were also deciphered to understand the 
emergence of multidrug-resistant diarrheal pathogens. Current findings will help in the establishment of key facts for active surveillance and management of diarrheal diseases as well as prioritizing strategies for combating drug resistance.

\section{MATERIALS AND METHODS}

\section{Materials}

The selective, differential, and routine culture media - thiosulfate citrate bile salt (TCBS) agar, MacConkey agar, glucose phosphate medium, triple sugar iron agar, Simmon's citrate agar, and peptone were purchased from Hi-Media Lab. Ltd., India. Commercially available antibiotic disks were also purchased from Hi-Media, Mumbai, India. Sodium chloride, Tris base, glacial acetic acid, ethylenediaminetetracetate, ethidium bromide, and other chemicals of analytical grade were purchased from Merck Pvt. Ltd. India. Polymerase chain reaction (PCR) Master Mix was purchased from ClonTech Takara, and DNA ladder of $100 \mathrm{bp}$ and $1 \mathrm{~kb}$ were bought from New England Biolabs. The primers used in the study were synthesized and procured from Integrated DNA Technology, India. Sequencing of amplified PCR products was performed commercially at Eurofins Genomics India Pvt Ltd.

\section{Study subject and sample collection}

During February 2013-April 2016, a total of 572 fecal specimens were collected from diarrheal patients admitted in Indira Gandhi Medical College, Shimla, and Government Zonal Hospital Solan, Himachal Pradesh. Diarrheal stool specimens were immediately transferred to the laboratory. The research protocols and methodology followed in the study were approved by the Institutional Ethics Committee (IEC/project no-04-2014). Patients enrolled in the study were from different age groups, and written informed consents were obtained before specimen collection. Clinical features such as frequency of diarrheal episodes, dehydration, fever, vomit, and abdominal pain were also documented as a part of clinical history.

\section{Culturing and biochemical characterization}

Collected stool specimens were initially screened for different bacterial and viral agents as per the standard microbiological protocols [21]. For Vibrio species, specimens were instantly inoculated into alkaline peptone water ( $\mathrm{pH} 8.5$ ) and enriched by incubating at $37^{\circ} \mathrm{C}$ for $16-18 \mathrm{~h}$. Following incubation, specimens were streaked onto TCBS agar and Mac-Conkey agar and incubated at $37^{\circ} \mathrm{C}$ for $24-48 \mathrm{~h}$. Yellow golden colonies from TCBS agar were chosen and preserved as glycerol stock at $-20^{\circ} \mathrm{C}$ for further analysis. Presumptive colonies from TCBS agar were subjected to a series of carbohydrate and amino acid utilization assays including indole, methyl red, Voges-Proskauer's, Simmon's citrate, and triple sugar iron agar test.

\section{Extraction of nucleic acid}

Biochemically characterized Vibrio isolates were further taken for molecular confirmation. DNA from bacterial isolates was extracted by colony boiling method [21]. In brief, 2-3 colonies from TCBS agar plate were suspended in $20 \mu \mathrm{l}$ nuclease-free water in PCR vial and heated at $95^{\circ} \mathrm{C}$ for $10 \mathrm{~min}$ in a water bath. Suspension was centrifuged at $3000 \mathrm{rpm}$ for $1 \mathrm{~min}$, and the supernatant was utilized as DNA template for PCR assays.

\section{Molecular characterization of Vibrio isolates}

The molecular characterization of Vibrio spp. was performed by PCR amplification of $16 S$ rRNA gene [22]. Primers used for molecular identification of Vibrio species included forward primer 5'- CGGTGAAATGCGTAGAGAT -3' and reverse primer 5'-TTACTAGCGATTCCGAGTTC-3' [22]. $2 \mu \mathrm{l}$ of DNA extracted by colony heat boiling was amplified in a $15 \mu \mathrm{l}$ PCR reaction mixture, $7.5 \mu \mathrm{l}$ of $\times 2$ PCR master mix, $0.25 \mu$ l of each primer, and nuclease-free water.

PCR amplification was accomplished using following conditions: 1 cycle of pre-denaturation at $95^{\circ} \mathrm{C}$ for $5 \mathrm{~min}$, followed by 35 cycles of final denaturation at $95^{\circ} \mathrm{C}$ for $30 \mathrm{~s}$, annealing at $48^{\circ} \mathrm{C}$ for $40 \mathrm{~s}$, extension at $72^{\circ} \mathrm{C}$ for $50 \mathrm{~s}$, and final extension at $72^{\circ} \mathrm{C}$ for $7 \mathrm{~min}$. A no template
PCR was run as a negative control for the reaction. The amplified PCR products were analyzed with ethidium bromide stained $1.5 \%$ agarose gel (in $\times 1$ TAE buffer) and visualized using UV transilluminator. The amplified PCR product of 16SrRNA gene was confirmed by sequencing.

\section{Antibiotic susceptibility tests}

Antimicrobial drug susceptibility assays were performed according to the Clinical Laboratory Standard Institute (CLSI) guidelines [23]. KirbyBauer's disk diffusion on Mueller-Hinton agar was used to evaluate antibiogram patterns against fifteen antibiotics: Cefepime (30 $\mu \mathrm{g})$, ceftazidime $(30 \mu \mathrm{g})$, ceftriaxone $(30 \mu \mathrm{g})$, imipenem $(10 \mu \mathrm{g})$, netilmicin (30 $\mu \mathrm{g})$, amikacin $(30 \mu \mathrm{g})$, kanamycin $(30 \mu \mathrm{g})$, tobramycin $(10 \mu \mathrm{g})$, streptomycin (10 $\mu \mathrm{g})$, ofloxacin (5 $\mu \mathrm{g})$, ciprofloxacin (5 $\mu \mathrm{g})$, levofloxacin (5 $\mu \mathrm{g})$, norfloxacin $(10 \mu \mathrm{g})$, nalidixic acid (30 $\mu \mathrm{g})$, and vancomycin (30 $\mu \mathrm{g}$ ). Results were interpreted according to manufacturer's instruction for Enterobacteriaceae family.

\section{Statistical analysis}

Determination of Vibrio-specific incidences among particular age group was accessed by Fisher's exact test. The statistical analysis was performed at 95\% confidence interval (CI), and odds ratios (OR) were calculated for Vibrio-infected population $(n=29)$ and Vibrio-negative population $(n=543)$. A p $\leq 0.05$ was considered as the positive level of significance for Fisher's exact test.

\section{RESULTS}

\section{Vibrio incidences among population}

From February 2013 to April 2016, a total of 572 fecal specimens were collected and screened for rotavirus, diarrheagenic E. coli and Vibrio species by biochemical and molecular methods to characterize these pathogens in regions of Himachal Pradesh. Collected specimens were from patients aged between 13 days and 85 years and grouped into five categories: Infants ( $0-2$ years), children (3-5 years), adolescent (6-17 years), adult (18-65 years), and elderly ( $>65$ years). Molecular characterization of biochemically confirmed Vibrio isolates showed amplification of Vibrio genus-specific 16S rRNA gene in 5\% ( $n=29 / 572)$ isolates (Fig. 1). Mixed enteric infection rates of Vibrio with E. coli and with rotavirus were observed to be $1.2 \%$ and $0.03 \%$, respectively. The amplified product of vibrio genus-specifi $16 \mathrm{SrRNA}$ gene [663bp] was confirmed by sequencing.

\section{Pattern of Vibrio infections among different age groups}

Incidences of Vibrio were analyzed among all five age groups, i.e., infants (0-2 years), children ( $3-5$ years), adolescents (6-17 years), adults (18-65 years), and elderly (>65 years) (Fig. 2). The highest frequency of Vibrio infection was observed in adolescents (7.3\%) and adult age $(6.6 \%)$ groups, while lowest infection rates observed among infants (2.9\%), followed by the elderly (3.7\%) and children population (4.4\%).

Fisher's exact test was calculated to Decipher highest risk age group among Vibrio-infected population (Table 1).

\section{Seasonal frequency of Vibrio incidences}

Screening of Vibrio species was carried out throughout the year to analyze season-wise distribution (Fig. 3). Season-wise classification of

Table 1: Identification of high-risk age group among diarrheal patients by Fisher exact test

\begin{tabular}{llll}
\hline Age groups & p value at $\mathbf{9 5 \%}$ CI & OR & Significance \\
\hline 0-2 years & 0.5896 & 0.7959 & NS \\
3-5 years & 1.0000 & 1.2 & NS \\
6-17 years & 0.9259 & 2.053 & NS \\
18- & 1.0000 & 1.842 & NS \\
65 years & & & \\
$>$ 65 years & Reference & & \\
& category & & \\
\hline
\end{tabular}

NS: Not significant, CR: Confidence interval, OR: Odd ratio 
months was performed according to Indian seasons: Summer (March to May), rainy (June to September), autumn (October to November), and winter (December to February). Highest incidences of Vibrio were observed for rainy season (34.3\%), followed by winter $(27.5 \%)$ and summer seasons (23.9\%). Among different age groups, infants were highly susceptible to Vibrio species during winter and summer seasons while no case was reported among infants in rainy season. For adolescent and adults, being the most active and productive group, highest infection was observed during rainy season, followed by winter and summer. Overall, seasonality peaks observed to have a rise in summer, reached at a maximum in rainy and a final decline during autumn.

\section{Antimicrobial resistance patterns of Vibrio species}

According to the Indian Council of Medical Research (ICMR) different

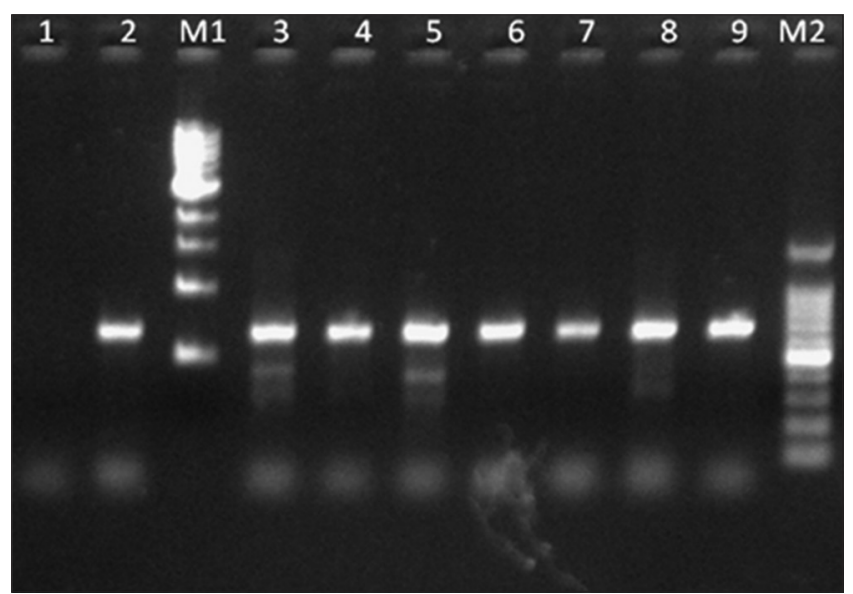

Fig. 1: Polymerase chain reaction (PCR) assay for characterization of Vibrio spp. through amplification of $16 \mathrm{~S}$ rRNA gene. Lane 1 shows a no template control. Lane 2 and lanes 3-9 show amplified PCR product for Vibrio genus-specific 16S rRNA gene (663bp). Lanes M1 and M2 show $1 \mathrm{~Kb}$ and 100 bp DNA ladder (NEB), respectively

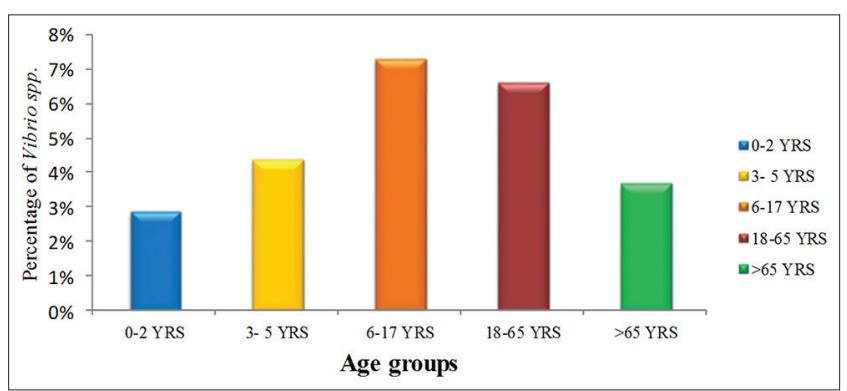

Fig. 2: Incidences of Vibrio spp. among different age groups of moderate-to-severe diarrhea-infected patients. 0-2 years: Infant, 3-5 years: Children, 6-17 years: Adolescent, 18-65 years: Adult, $>65$ years: Elderly population

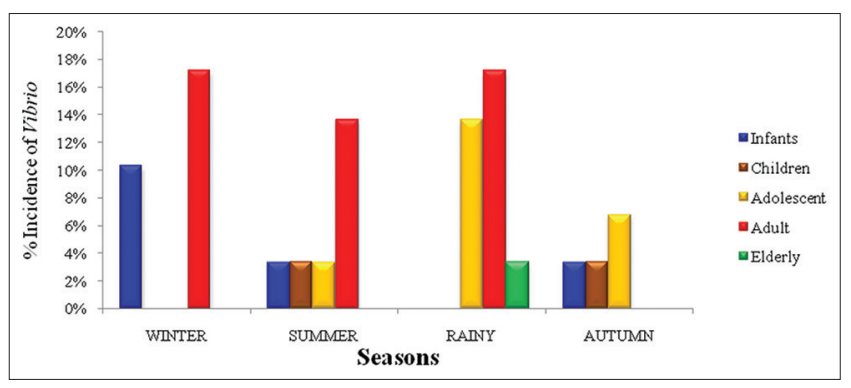

Fig. 3: Seasonal trends of Vibrio species incidences among different age categories of diarrheal patients antibiotic classes, tetracycline, $\beta$-lactam, and quinolones are prescribed for the treatment of severe diarrhea cases. Treatment regimen involving norfloxacin, nalidixic acid, cotrimoxazole tetracycline, and ampicillin are specific for Vibrio-mediated diarrhea [24]. Other antibiotics were also screened in the study to investigate the common resistance profiles of Vibrio spp. Accordingly, Kirby-Bauer's disk diffusion test was performed against fifteen different antibiotics (Fig. 4a-d).

In $\beta$-lactam class, alarming resistance levels were analyzed for ceftazidime (65.5\%), cefipime (62\%), and ceftriaxone $(55.1 \%)$ antibiotics (Fig. 4a). The proportion of intermediate isolates was found from $6.8 \%$ to $20.6 \%$ for all $\beta$-lactam antibiotics. However, lowest resistance was observed for imipenem (13.8\%).

Among aminoglycosides, highest frequency of resistance was observed against streptomycin (34.4\%) and kanamycin (31\%) (Fig. 4b). Percentage of intermediate isolates for aminoglycosides group ranged from $6.8 \%$ to $31 \%$. Vibrio spp. exhibited minimum resistance levels for netilimicin $(6.8 \%)$, followed by tobramycin $(13.7 \%)$ and amikacin (20.6\%).

For quinolones, alarming resistance rates were observed for nalidixic acid $(72.4 \%)$, followed by ofloxacin (37.9\%), ciprofloxacin $(37.9 \%)$, and norfloxacin (27.4\%) (Fig. 4c). Vibrio spp. exhibited least resistance against levofloxacin (30.1\%). Proportions of intermediate isolates for quinolones were observed to range between $3.4 \%$ and $20.6 \%$.

Overall, Vibrio isolates were most sensitive for imipenem, netilmicin, tobramycin, levofloxacin, and norfloxacin, and hence, these antibiotics could be priority antibiotics of choice for treatment of Vibrio infections. Moreover, continuous monitoring of antibiotic resistance is required to access $\beta$-lactam resistance trends as third-generation cephalosporinresistant Vibrio incidences are rare and anxiously alarming [11,14].

\section{DISCUSSION}

Diarrhea is a gastrointestinal illness of multietiology and multifactorial nature with high morbidity and mortality incidences [1]. In the past few years, increased incidences of Vibrio have been reported from various parts of the globe [25] with coastal regions more prone to this infectious disease $[18,25]$. Historically, Vibrio has caused many pandemic and epidemics along with several explosive outbreaks which continue to thrive in regions with contaminated water and suboptimal hygiene practices [26-28]. Diarrhea-associated morbidity and mortality rates are quite higher in Indian sub-continent than the rest parts of world [1,26], and many parts of country are endemic for cholera disease. In Southeast Asian region, India and Bangladesh have reported highest Vibrio infections, and in the past few years, threefold increase in infection rates has been reported [25]. Himachal Pradesh is a Northern hilly state of India with varied geographical conditions, and comprehensive studies elucidating Vibrio species incidences and antimicrobial surveillance are limited.

In the previous studies, we investigated the role and incidences of viral pathogens as etiological agents of diarrhea in the region [20]. The present study was focused to explore infection rates of Vibrio spp. in the state. Antimicrobial susceptibility patterns of Vibrio isolates were also determined according to standards of CLSI against commonly utilized antibiotics. The population under the study comprised of moderate-tosevere diarrhea patients admitted to regional and tertiary care hospital settings of Himachal Pradesh.

Standard biochemical assays and molecular methods were utilized to investigate incidences of Vibrio in diarrhea cases. Initially, Vibrio isolates were biochemically confirmed by IMViC and triple sugar iron agar tests followed by molecular characterization. By amplification of Vibrio genus-specific 16S rRNA gene, infection rate of Vibrio was found to be $5 \%$ (29/572) among diarrheal population (Fig. 1). In a previous study, Bora et al. reported $16.5 \%$ of Vibrio infections in an outbreak from remote areas of Himachal Pradesh [29]. In addition, such higher 


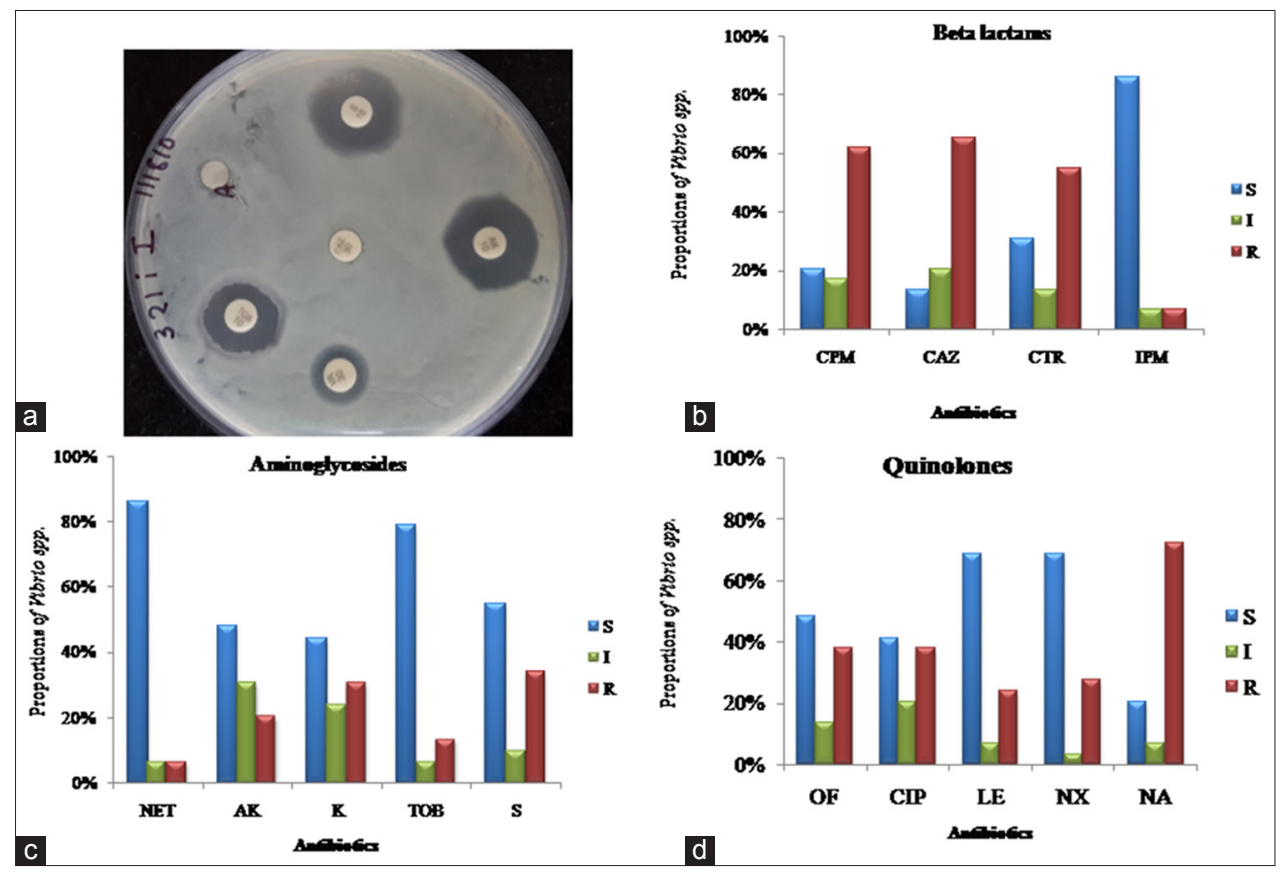

Fig. 4: Antibiotic susceptibility patterns of Vibrio spp. (a) antibiotic susceptibility performed by Kirby-Bauer's disk diffusion method. Proportions of sensitive (S), intermediate (I), and resistant (R) phenotypes for $\beta$ lactams (b), aminoglycosides (c), and quinolones (d). CPM - cefepime, CAZ - ceftazidime, CTR - ceftriaxone, IPM - imipenem, NET - netilmicin, AK - amikacin, K - kanamycin, TOB - tobramycin, $S$ - streptomycin, OF - ofloxacin, CIP - ciprofloxacin, LE - levofloxacin, NX - norfloxacin, and NA - nalidixic acid

Vibrio spp. incidences have also been reported from surrounding areas of state [16,17]. Another study deciphered presence of higher proportion of Vibrio spp. in water samples collected from different river systems [30]. Similarly, Vibrio incidence rates of $2-4 \%$ have also been reported from surroundings of Himachal Pradesh [31-33]. In comparison to our observation, higher Vibrio infection rates ranging from $10 \%$ to $15 \%$ are reported from different parts of India $[34,35]$. Furthermore, surveillance studies from Bangladesh reported up to $18 \%$ of Vibrio infections among diarrheal populations [3,37]. Overall, our study depicts low levels of Vibrio spp. incidences, and similar trends are observed for active surveillance studies in the country.

Incidences of Vibrio were found to be higher among adolescent and adult population, and similar observations were found in other report [38]. However, children $<5$ years age group (0-2 and 3-5 years) also suffered with similar infection rates as that of adult. CDC weekly morbidity and mortality reports also found children $<5$ years as most vulnerable group to Vibrio infection [3].

Seasonal variations for Vibrio incidences are driven by various factors such as recurrent floods stagnant water and suboptimal hygienic conditions $[39,40]$. Annually, Himachal Pradesh is fed by snow and rainfall; however, owing to its mountainous slopes, water is drained into two main basins: Indus and Gangetic river systems. Uniform seasonal distribution of Vibrio spp. was seen throughout the years; however, slight peaks were observed during rainy, winter, and summer seasons. Similar seasonal trends have been observed from coastal area, but frequency is much higher as compared to current study [27,37-41]. This may be due to lack of stagnant water reservoirs in the region and direct drainage of rain water into major river systems.

Vibrio is known to cause life-threatening complications, if left untreated. Therapeutic approaches involving rehydration therapy combined with antibiotics reduces duration of infection and fatality rates. So far, antibiotic resistance pattern have been utilized as key epidemiological and phenotypic markers to trace the evolution of outbreak strains. Hence, it is imperative to monitor resistance levels in Vibrio pathogen to regulate proper treatment regimen. Although screening for all antibiotics utilized in the current study is not recommended by ICMR guidelines [24], the current study also elucidated common antibiotic patterns exhibited by Vibrio as reported in other studies [42].

Vibrio strains showed greatest sensitivity levels against imipenem (86.2\%), followed by netillin, tobramycin, levofloxacin, ofloxacin, amikacin, and kanamycin (Fig. 4a-d). Similar findings have also been reported from other parts of country [27,31-33]. Moreover, our study showed current treatment regimen effective against Vibrio isolates.

Vibrio strains showed rising levels of resistance against beta-lactam group (cefepime, ceftazidime, and ceftriaxone) and nalidixic acid. Vibrio strains having resistance against cephalosporins have been reported scarcely [15], while the current study elicited alarming resistance rates against cephalosporin class in the region. The previous studies reported very higher levels of resistance against nalidixic acid, streptomycin, and ciprofloxacin antibiotics $[31,32,43,44]$; however, our study implicated relatively low levels of resistance against these antibiotics.

Overall, our study elucidated lower incidences of Vibrio species as compared to other parts of country. Our findings also provide new insight regarding incidences and prevalence of Vibrio spp. and its seasonal distribution and resistance management. For developing nations like India, strategic management of infectious diarrhea should be accelerated at a great pace through continuous and active surveillance of etiological agents to reduce disease-associated morbidity and mortality rates.

\section{REFERENCES}

1. Kotloff KL, Nataro JP, Blackwelder WC, Nasrin D, Farag TH, Panchalingam S, et al. Burden and aetiology of diarrheal disease in infants and young children in developing countries (the global enteric multicenter study, GEMS): A prospective, case-control study. Lancet 2013:382:209-22.

2. World Health Organization. Flooding and communicable diseases fact sheet. Wkly Epidemiol Rec 2005;80:21-8.

3. Crim SM, Griffin PM, Tauxe R, Marder EP, Gilliss D, Cronquist AB, et al. Preliminary incidence and trends of infection with pathogens 
transmitted commonly through food - Foodborne diseases active surveillance network, 10 US sites, 2006-2014. Morb Mortal Wkly Rep 2015;64:495-9.

4. Ali M, Lopez A, You Y, Kim Y, Sah B, Maskery B, et al. The global burden of cholera. Bull World Health Organ 2012;90:209-18.

5. Available from: http://www.emro.who.int/surveillance-forecastingresponse/surveillance-news/cholera-cases-update-from-yemen-17november-2016.html.

6. Sarkar BL, Kanungo S, Nair GB. How endemic is cholera in India? Indian J Med Res 2012;135:246-8.

7. Kaper JB, Morris JG, Levine MM. Cholera. Clin Microbiol Rev 1998;8:48-86.

8. Nair GB, Ramamurthy T, Bhattacharya SK, Mukhopadhyay AK, Garg S, Bhattacharya MK, et al. Spread of Vibrio cholerae 0139 Bengal in India. J Infect Dis 1994;169:1029-1034.

9. Almagro-Moreno S, Pruss K, Taylor RK. Intestinal colonization dynamics of Vibrio cholerae. PLoS Pathog 2015;11:e1004787.

10. Jiang S, Chu W, Fu W. Prevalence of cholera toxin genes (ctxA and zot) among non-O1/O139 Vibrio cholerae strains from Newport bay, California. Appl Environ Microbiol 2003;69:7541-4.

11. Li X, Wang D, Li B, Zhou H, Liang S, Ke C, et al. Characterization of environmental Vibrio cholerae serogroups O1 and O139 in the Pearl river estuary, China. Can J Microbiol 2016;62:139-47.

12. Qureshi AA, Omer S, Kumar KE, Bhajipale NS. Probiotics in diarrhea: Myths and facts. Int J Pharm Pharm Sci 2010;2:23-8.

13. Shariff WR, Karigar A, Sikarwar MS. Evaluation of antidiarrheal activity of crataeva nurvala root bark in experimental animals. Int J Pharm Pharm Sci 2010;2 Suppl 1:158-61.

14. World Health Organization. UNICEF. Geneva: Clinical Management of Acute Diarrhea: WHO; 2004

15. Mandal J, Dinoop KP, Parija SC. Increasing antimicrobial resistance of Vibrio cholerae OI biotype EI tor strains isolated in a tertiary-care centre in India. J Health Popul Nutr 2012;30:12-6.

16. Jaiswal A, Sarkar S, Das P, Nandy S, Koley H, Sarkar B, et al. Trends in the genomic epidemiology of Vibrio cholerae O1 isolated worldwide since 1961. Int J Antimicrob Agents 2015;46:460-4.

17. Mutreja A, Kim DW, Thomson NR, Connor TR, Lee JH, Kariuki S, et al. Evidence for several waves of global transmission in the seventh cholera pandemic. Nature 2011;477:462-5

18. Taneja N, Sangar G, Chowdhury G, Ramamurthy T, Mishra A, Singh M, et al. Molecular epidemiology of Vibrio cholerae causing outbreaks and sporadic cholera in Northern India. Indian J Med Res 2012;136:656-63.

19. Mishra A, Taneja N, Sharma RK, Kumar R, Sharma NC, Sharma M, et al. Amplified fragment length polymorphism of clinical and environmental Vibrio cholerae from a freshwater environment in a cholera-endemic area, India. BMC Infect Dis 2011;11:249.

20. Jain S, Thakur N, Grover N, Vashistt J, Changotra H. Prevalence of rotavirus, Norovirus and Enterovirus in diarrheal diseases in Himachal Pradesh, India. Virusdisease 2016;27:77-83

21. Panchalingam S, Antonio M, Hossain A, Mandomando I, Ochieng B, Oundo J, et al. Diagnostic microbiologic methods in the GEMS-1 case/ control study. Clin Infect Dis 2012;55 Suppl 4:S294-302.

22. Lipp EK, Rivera IN, Gil AI, Espeland EM, Choopun N, Louis VR, et al. Direct detection of Vibrio cholerae and ctxA in Peruvian coastal water and plankton by PCR. Appl Environ Microbiol 2003;69:3676-80.

23. Patel JB, Cockerill FR, Bradford PA, Eliopoulos GM, Hindler JA. Jenkins SG, et al. Performance Standards for Antimicrobial Susceptibility Testing; Twenty-Fifth Informational Supplement 2015;3:1-240

24. Available from: http://www.ricmr.nic.in/Publications/SOP/SOP Bacteriology.pdf.

25. World Health Organization. Global Health Observatory (GHO): Number of Reported Cholera Cases. Available from: http://www.who. int/gho/epidemic diseases/cholera/cases text/en.

26. Mukhopadhyay AKK, Takeda Y, Balakrish Nair G. Cholera outbreaks in the el tor biotype era and the impact of the new el tor variants. Curr Top Microbiol Immunol 2014;379:17-47.

27. Pal BB, Khuntia HK, Nayak SR, Mohanty A, Biswal B. Vibrio cholerae O1 ogawa strains carrying the ctxB7 allele caused large cholera outbreak during 2014 in the tribal areas of Odisha, India. Jpn J Infect Dis 2017;70:549-55.

28. Available from: https://www.cdc.gov/cholera/asia/index.html\#one last.

29. Bora D, Dhariwal AC, Jain DC, Sachdeva V, Vohra JG, Prakash RM, et al. V. cholerae 01 outbreak in remote villages of Shimla district, Himachal Pradesh, 1994. J Commun Dis 1997;29:121-5.

30. Kumar P, Shankar U, Paul P. Identification the presence of Vibrio species by TCBS media in different water samples collected from different locations. Int J Sci Res 2014;26:346-51.

31. Mala E, Oberoi A, Alexander VS. Vibrio isolates from cases of acute diarrhea and their antimicrobial susceptibility pattern in a tertiary care hospital. Int J Basic Appl Sci 2014;3:35-37.

32. Kumar A, Oberoi A. Vibrio isolates from cases of acute diarrhea and their antibiotic susceptibility pattern in a tertiary care hospital of Punjab. CHRISMED J Health Res 2014;1:254

33. Uppal B, Mehra B, Panda PS, Kumar SK. Antimicrobial susceptibility profile of Vibrio cholerae strains isolated at a tertiary care medical centre in New Delhi, India. Int J Commun Med Pub Health 2017;4:868-71.

34. Dutta D, Chowdhury G, Pazhani GP, Guin S, Dutta S, Ghosh S, et al. Vibrio cholerae non-O1, non-O139 serogroups and cholera-like diarrhea, Kolkata, India. Emerg Infect Dis 2013;19:464-7.

35. Chhotray GP, Ranjit MR, Pal BB, Meher PK, Khuntia HK. Incidence and Molecular Analysis of Vibrio cholerae among some primitive Tribes in Odisha, India. Int J Curr Microbiol Appl Sci 2017;6:51-61.

36. Paul RC, Faruque AS, Alam M, Iqbal A, Zaman K, Islam N, et al. Incidence of severe diarrhoea due to Vibrio cholerae in the catchment area of six surveillance hospitals in Bangladesh. Epidemiol Infect 2016;144:927-39.

37. Rashed SM, Hasan NA, Alam M, Sadique A, Sultana M, Hoq MM, et al. Vibrio cholerae $\mathrm{O} 1$ with reduced susceptibility to ciprofloxacin and azithromycin isolated from a rural coastal area of Bangladesh. Front Microbiol 2017;8:252.

38. Thapa Shrestha U, Adhikari N, Maharjan R, Banjara MR, Rijal KR, Basnyat SR, et al. Multidrug resistant Vibrio cholerae $\mathrm{O} 1$ from clinical and environmental samples in Kathmandu city. BMC Infect Dis $2015 ; 15: 104$

39. Ramamurthy T, Yamasaki S, Takeda Y, Nair GB. Vibrio cholerae O139 Bengal: Odyssey of a fortuitous variant. Microb Infect 2003;5:329-44

40. Mookerjee S, Jaiswal A, Batabyal P, Einsporn MH, Lara RJ, Sarkar B, et al. Seasonal dynamics of Vibrio cholerae and its phages in riverine ecosystem of Gangetic west Bengal. Cholera paradigm. Environ Monit Assess 2014;186:6241-50.

41. Deepanjali A, Kumar HS, Karunasagar I, Karunasagar I. Seasonal variation in abundance of total and pathogenic Vibrio parahaemolyticus bacteria in oysters along the southwest coast of India. Appl Environ Microbiol 2005;71:3575-80.

42. Baron S, Lesne J, Jouy E, Larvor E, Kempf I, Boncy J, et al. Antimicrobial susceptibility of autochthonous aquatic Vibrio cholerae in Haiti. Front Microbiol 2016;7:1671.

43. Garg P, Chakraborty S, Basu I, Datta S, Rajendran K, Bhattacharya T, et al. Expanding multiple antibiotic resistance among clinical strains of Vibrio cholerae isolated from 1992-1997 in Calcutta, India. Epidemiol Infect 2000;124:393-9.

44. Ghosh R, Sharma NC, Halder K, Bhadra RK, Chowdhury G, Pazhani GP, et al. Phenotypic and genetic heterogeneity in Vibrio cholerae O139 isolated from cholera cases in Delhi, India during 2001-2006. Front Microbiol 2016;7:1250. 